\title{
¿Qué clase de criaturas somos?
}

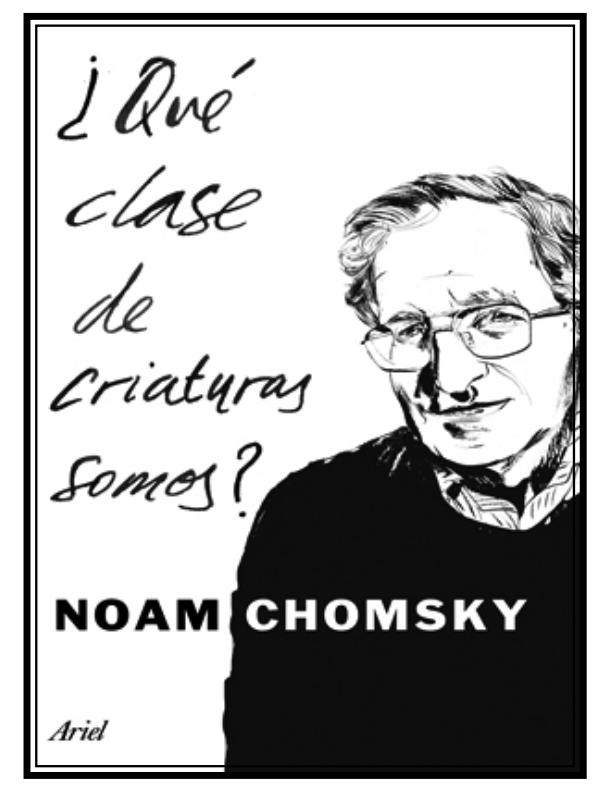

Noam Chomsky (2017). ¿Qué clase de criaturas somos? Barcelona: Editorial Planeta. Versión española de Jorge Paredes y con un prólogo de Akeel Bilgrami.

Este libro presenta las reflexiones de toda una vida de un científico del lenguaje sobre las conclusiones más amplias de su trabajo de investigación. El título de esta obra, ¿Qué clase de criaturas somos?, expresa lo amplias que se supone que deben ser dichas conclusiones. Abarcan una impresionante serie de campos: lingüística teórica, ciencia cognitiva, filosofía de la ciencia, historia de la ciencia, biología evolutiva, metafísica, teoría del conocimiento, filosofía del conocimiento y de la mente, filosofía moral y política y, aunque brevemente, el ideal de la educación humana.

El capítulo 1 presenta, con precisión y claridad, las propias ideas fundamentales de Noam Chomsky acerca de la lingüística teórica y la ciencia cognitiva (campos en los que ha desempeñado un papel absolutamente esencial), exponiendo los avances logrados a lo largo de los años, pero mucho más intensamente cómo tienen que llevarse a cabo esas tentativas de avance y cómo continúa pendiente una enorme cantidad de trabajo, incluso en los ámbitos de estudio más fundamentales. También recoge los cambios de mentalidad a lo largo de los años, y algunos de los más impactantes de ellos han tenido lugar en la última década.

El capítulo comienza planteando la pregunta anunciada en su título: “Qué es el lenguaje?”. Nos corresponde a nosotros hacer la pregunta porque, si no tenemos claro qué es el lenguaje, no sólo no obtendremos las respuestas correctas a otras preguntas sobre diversos aspectos concretos del lenguaje (puede que ni siquiera seamos capaces de formular correctamente esas preguntas específicas), sino que no nos aproximaremos a investigar o siquiera a especular de manera plausible acerca de la base biológica y el origen evolutivo del lenguaje.

Una tradición que se remonta a Galileo y Descartes identificaba la característica más fundamental del lenguaje, cuya articulación más explícita fue posteriormente la de Humboldt: "La lengua se enfrenta en el sentido más genuino con un dominio infinito y sin fronteras, el conjunto de todo lo pensable. Eso le obliga a hacer un uso infinito de medios finitos, cosa que 
le es posible en virtud de la fuerza que engendra la identidad de las ideas y el lenguaje" ${ }^{\prime 1}$. También se cita a Darwin repitiendo esto mismo de una manera más elemental en el contexto del interés evolutivo por el lenguaje: “Los animales inferiores difieren del hombre únicamente en su casi infinita capacidad de asociar los más diversos sonidos e ideas". Vale la pena señalara que aquí hay tres características fundamentales observadas por Humboldt y Darwin. Primera, la referencia a una capacidad infinita sobre una base finita; segunda, la conexión entre ideas y sonido, y tercera, la conexión entre lenguaje y pensamiento. Todas ellas se agrupan en lo que Chomsky define al principio como la Propiedad Básica del Lenguaje: "Cada lengua presenta una serie ilimitada de expresiones estructuradas jerárquicamente que reciben interpretaciones en dos interfaces, sensoriomotora por lo que respecta a la externalización y conceptualintencional por lo que respecta a los procesos mentales". El elemento estructural jerárquico se refiere a la primera característica; la interfaz sensoriomotora se refiere a la segunda, y la interfaz conceptual-intencional, a la tercera.

Lo que explicará esta Propiedad Básica es un proceso computacional. La importancia filosófica de esto es doble: una teoría del lenguaje es necesariamente una gramática generativa, y la teoría hace referencia necesariamente a un objeto que poseen los humanos, intrínseco al sujeto individual y a su mentalidad (esto es, elementos intensionales): No se trata de una teoría sobre afirmaciones externalizadas ni, por tanto, sobre un fenómeno social. La nomenclatura para plasmar esta última distinción entre lo que es individual/interno/intensional y lo que es externalizado/social es lenguaje I y lenguaje E respectivamente. Los lenguajes I son los únicos que pueden ser objeto de estudio científico; los lenguajes $\mathrm{E}, \mathrm{no}^{2}$. $\mathrm{Y}$, a pesar de que dicho estudio acabará teniendo una explicación biológica, hasta ese momento la ciencia capta el fenómeno a un nivel de abstracción de la biología y hace referencia al nivel cognitivo de la capacidad computacional que satisface la Propiedad Básica $^{3}$. Una tarea distinta y más general es la de descubrir las características comunes que subyacen en todos los lenguajes, la cual, una vez más, viene determinada por las propiedades biológicas de las cuáles están dotados los seres humanos (tema cuya gran importancia para la cognición en general se trata de nuevo en el capítulo 2). Esta tarea más genérica se emprende con vistas a descubrir los atributos biológicos que determinan qué sistemas generativos pueden funcionar como lenguajes I. Dicho de otro modo, ¿cuáles son los posibles lenguajes humanos?

A continuación, Chomsky señala que, en cuanto se llevó a cabo un estudio serio de las gramáticas generativas en relación con la Propiedad Básica del lenguaje, surgieron algunos rompecabezas sorprendentes, con consecuencias trascendentales. Uno es la "dependencia estructural" de las operaciones lingüísticas: en todas las construcciones, en todos los lenguajes, esas operaciones dependen invariablemente de la distancia estructural en lugar de la idea mucho más sencilla desde un punto de vista computacional de la distancia lineal. Las personas que aprenden una lengua saben esto automáticamente, sin formación. Esto viene respaldado por la neurociencia y la psicología experimental. El resultado es consecuencia

1 Para todas las referencias, véanse los capítulos de la que se extraen las citas. Sobre la relación entre lenguaje e ideas, Chomsky, aunque ahora cree estar más cerca de lo que creía en su día, es necesario afirmar la existencia entre ambos de algo tan contundente como la "identidad", tal como hace Humboldt. Descartes y Darwin que también aparecen en la discusión de Chomsky sobre la relación, no llegaron tan lejos.

2 Aunque Chomsky menciona los lenguajes E en contraposición a los lenguajes I, duda de la coherencia de la idea misma y, por tanto, que existan. En varios de sus ensayos se muestra crítico con los presupuestos más básicos de los filósofos acerca de su coherencia a la hora de explicarlos.

$3 \mathrm{Al}$ reivindicar el estudio a nivel abstracto con vistas a una explicación eventual en términos cerebrales, Chomsky cita algunos avances que podrían haber logrado en la búsqueda de los fundamentos biológicos, pero también cita cómo es posible que haya algunos presupuestos fundamentalmente erróneos por científicos expertos en el cerebro acerca de cuál es el objeto de estudio. Sobre este último punto, véase su referencia a la obra de Charles Gallistel en el capítulo 2. 
de asumir que el orden simplemente no está disponible en las operaciones que generan las expresiones estructuradas interpretadas en la interfaz conceptual internacional, para el pensamiento y la organización de la acción. Esto se desprende, a su vez, de la asunción absolutamente natural de que los lenguajes I son sistemas generativos basados en la operación computacional más elemental, la cual carece de orden. Estas y numerosas consideraciones más proporcionan pruebas sustanciales de que el orden lineal está subordinado al lenguaje, no se encuentra en el centro de la sintaxis y la semántica. Lo mismo sucede con los diversos acuerdos del lenguaje de signos, el cual ahora sabemos que es extraordinariamente parecido al lenguaje oral en cuanto a estructura, adquisición, uso e incluso representación neuronal. Presumiblemente, estas propiedades externas reflejan condiciones impuestas por el sistema sensoriomotor. La opción de utilizar el orden lineal ni siquiera se plantea para quien aprende una lengua. El orden lineal y otros argumentos son relevantes para lo que se oye -es decir, lo que se exterioriza-, no para lo que se piensa, que es interno.

A continuación, señala que estas conclusiones cuadran con lo poco que se sabe acerca del origen del lenguaje. El sistema sensoriomotor, "al parecer existía mucho antes de la aparición del lenguaje" y parece haber muy poca adaptación específica al lenguaje. Propiedades cognitivas mucho más profundas que las poseídas por los simios, o presumiblemente por homínidos o humanos, son intrínsecas al lenguaje. Los simios poseen sistemas gestuales adecuados para hacer signos y sistemas auditivos adecuados para la percepción del habla; sin embargo, a diferencia de los bebés humanos, interpretan el habla como simple ruidos $y$, ni siquiera tras un entrenamiento exhaustivo, pueden alcanzar los rudimentos del lenguaje de signos humano. Aristóteles dijo que el lenguaje es "sonido con significado", pero estas consideraciones que acabamos de resumir insinúan, según Chomsky, que las propiedades del eslogan pueden invertirse y que el lenguaje se entendería mejor como "significado con sonido". En caso de que esto parezca platonista (algo que fue fervientemente divulgado por Jerrold Katz), hay que recordar que, en este caso, para Chomsky, "significado" debe interpretarse como una categoría absolutamente psicológica (en definitiva biológica) y, por tanto, no puede cosificarse en absoluto en términos platónicos.

Tales conclusiones, a su vez, avivan la antigua afirmación de Chomsky de que el lenguaje no ha de entenderse como lo entienden en todas partes los filósofos, antropólogos, etc., como, de alguna manera definitoria, ligado a la comunicación. Si la exteriorización del lenguaje es secundaria y el vínculo del lenguaje con el pensamiento es fundamental, la comunicación no puede ser clave para responder a la pregunta formulada en este capítulo: “QQué es el lenguaje?”. De hecho, como él dice, hay motivos para pensar que la mayor parte del lenguaje/pensamiento no se exterioriza en absoluto. Si creemos firmemente que el lenguaje no ha sido diseñado por los seres humanos, sino que forma parte de sus características biológicas, para hacer del lenguaje un objeto de estudio, ya sea científico o filosófico, debería haber un cambio considerable en nuestro enfoque metodológico.

La cita de Darwin a la que Chomsky hace referencia con aprobación dice que lo que resulta fundamental acerca del lenguaje es una "capacidad de asociar los sonidos e ideas más diversas". Salvo por el hecho de que, como hemos mencionado, el sonido (junto a otras formas de exteriorización) ha sido degradado, la propia exposición teórica de Chomsky acerca de la Propiedad Básica parte de las palabras de Darwin para su argumentación...si bien tal vez no de las palabras exactas, ya que "asociar" no es la forma más precisa de describir la operación central planteada en su exposición. La asociación tiene lugar, al fin y al cabo, incluso en el condicionamiento clásico (campana, comida) y es bien conocido que Chomsky ha rechazado las explicaciones conductuales del lenguaje. Por otra parte, las asociaciones entre dos objetos, tal como entienden la asociación incluso los psicólogos no conductuales, pueden implicar que el orden de los objetos es importante de un modo en que el mucho mayor peso es concedido a la forma para la interpretación semántica de la interfaz conceptual-intensional (en lugar de la interfaz sensoriomotora) establece que no lo 
es. De modo que, dejando de lado el equívoco término asociar para centrarse en lo que quiere decir el propio Darwin, lo que Chomsky tiene en mente es conceder especial importancia al hecho de que nuestra capacidad de "agrupar" ideas y elementos sintácticos nos hace únicos. Y esta concepción fundamental del lenguaje se refleja en la explicación teórica de la Propiedad Básica, en la cual a la operación determinante se la denomina Ensamble, la cual puede actuar externamente sobre dos objetos diferenciados para crear otro, o puede actuar internamente desde un objeto para crear otro, creando automáticamente la propiedad generalizada del "desplazamiento" (frases oídas en un sitio pero también entendidas en otro diferente) de manera apropiada para una interpretación semántica compleja.

Se los denomina Ensamble externo e interno, respectivamente, y el respeto por la sencillez en el método científico, aplicable tanto en la lingüística como en cualquier otro campo, determina que disponemos de la capacidad básica operativa que fundamenta la Propiedad Básica. Trabajando sobre algunos ejemplos para mostrar cómo el diseño del lenguaje está a su nivel óptimo si nos atenemos a estos requisitos metodológicos, Chomsky presenta cambios en su propia visión, como por ejemplo respecto al fenómeno del "desplazamiento" que en su día consideraba una "imperfección", mientras que ahora, si nos atenemos a las asunciones metodológicas más sencillas tal como acabamos de mencionar, es algo natural.

El capítulo concluye con un audaz intento de explotar estos últimos puntos metodológicos para unir dos cuestiones aparentemente dispares: ¿Qué explicación deberíamos darle a la Propiedad Básica? ¿Cómo y cuándo surgió el lenguaje? Esta confluencia de la sencillez de las asunciones a la hora de explicar la Propiedad Básica y la consiguiente afirmación del diseño óptimo del lenguaje puede contribuir a fundamentar la hipótesis más plausible según las escasas pruebas de que disponemos sobre el origen del lenguaje: que el lenguaje no surgió de manera gradual, sino de repente (y en una época relativamente reciente). Ahora es posible especular que un "gran salto adelante" de ese calibre tal vez fue provocado por una "leve reprogramación del cerebro [que] generó el Ensamble, de manera natural, de la manera más sencilla, proporcionando la base para el pensamiento ilimitado y creativo", del que se carecía hasta entonces.

El capítulo 2, “QQué podemos entender?”, combina algunas de esas conclusiones desarrollando en primer lugar otro tema fundamental de la obra de Chomsky: los límites de la cognición humana.

Existe una locución que todos hemos utilizado con frecuencia: "el alcance y los límites de...". Chomsky se lo toma en serio y da un giro determinante al desarrollar la visión de nuestras capacidades cognitivas, Esas capacidades, cuyo alcance es mayor y más profundo que las de cualquier otra criatura que conozcamos, son así en parte porque también están sometidas a límites, límites intrínsecos a nuestra naturaleza o, como sugiere el título del libro, a la clase de criaturas que somos; concretamente, al hecho de que nuestras capacidades cognitivas tengan una base biológica.

Implícitamente, nos topamos con este punto en el capítulo, aunque ahí se limita a la capacidad humana para el lenguaje. La explicación teórica del lenguaje ofrecida allí, presupone esta idea de límites, es decir, presupone que estamos dotados de estructuras innatas que nos conceden la capacidad exclusiva para el lenguaje, estructuras que, al mismo tiempo limitan lo que el lenguaje es para nosotros y qué lenguas posibles hay. El término técnico "GU" se refiere a la caracterización de esas estructuras innatas y es dentro del marco de alcance y los límites impuestos por esta dotación genética donde se explica el lenguaje como una capacidad computacional en la explicación generativa resumida anteriormente.

Lo cierto es que el lenguaje simplemente es un caso especial de una serie absolutamente general de alcances y límites que proceden del hecho de ser criaturas biológicas. Aparentemente, la idea no genera controversia por lo que respecta 
a la capacidad física: lo que nos hace aptos para caminar nos limita, ya que no estamos preparados para reptar como serpientes. Chomsky considera que es un prejuicio negar que lo que es obvio en el caso de esas capacidades físicas no es obvio (como indicarían las incesantes controversias en torno a las ideas innatas) en caso de las capacidades cognitivas. Poseer algunas capacidades cognitivas significa necesariamente que se puede carecer de otras capacidades cognitivas, capacidades cognitivas que podrían poseer otra clase de sujetos con cerebro. Únicamente si ignoramos el factor biológico a la hora de estudiar la cognición humana podemos plantearnos negar esos límites. El capítulo 2 examina el tema de esos límites sobre nuestras capacidades cognitivas de manera bastante general, más allá del ámbito concreto del lenguaje, a pesar de volver en varios puntos a extraer de nuevo conclusiones sobre el lenguaje. Examina el resultado metodológico de esta idea de límites cognitivos evocando una distinción planteada por Chomsky hace casi cinco décadas entre "problemas" y "misterios", haciendo referencia a la concepción de Peirce del método y el crecimiento científico que apela al concepto de abducción, el cual pone límites a lo que considera "hipótesis admisibles", sostiene que las estructuras innatas determinadas por nuestro legado genético ponen límites a las preguntas que podemos plantear. Las preguntas que podemos formular de manera controlada se llaman "problemas", pero, dados los límites dentro de los cuales es posible su formulación, habrá cosas que se escapen a nuestras capacidades cognitivas; en la medida en que podamos concebirlas, dados nuestros marcos conceptuales y conocimientos, seremos incapaces de formularlas de una manera en que pueda llevarse a cabo una investigación científica controlable. A esto lo denomina "misterios". El título de este libro, ¿Qué clase de criaturas somos?, se refiere directamente a esto, ya que otros tipos de criaturas, con un legado biológico distinto al nuestro, pueden ser capaces de formular problemas que para nosotros siguen siendo un misterio. Así, para Chomsky, si bien no para Peirce (el cual, al hablar de hipótesis admisibles otorga un papel menos determinante al hecho de que seamos criaturas biológicas) $)^{5}$. La distinción entre "problemas" y "misterios" tiene relación con el organismo. Es una parte muy importante de esta evaluación metodológica que deberíamos aprender a suavizar con el hecho de nuestros límites cognitivos y los "misterios" que nos obligan inevitablemente a reconocer. El último capítulo de este volumen, "Los misterios de la naturaleza", recorre momentos vitales de la historia de la ciencia para extraer esta lección metodológica.

Un momento crucial es aquel en que Newton derogó las creencias de la mecánica de contacto de la primera ciencia moderna anterior a él y planteó una idea de gravedad que echó por tierra las nociones anteriores de materia, movimiento y causalidad, las cuales eran afirmaciones de nuestra concepción basada en el sentido común (probablemente determinada por los límites cognitivos de nuestra biología) del mundo de los objetos. Chomsky señala que con Newton surgió un nuevo marco en el que -a tenor de esos límites- se estaba planteando algo inconcebible. El propio Newton lo reconoció, calificándolo de absurdo y, desde Newton, nadie ha hecho nada por cambiar las cosas en este sentido. Por el contrario, lo absurdo simplemente se ha incorporado a nuestra imagen científica del mundo. Newton nunca permitió que eso lo desanimara, elaborando leyes explicativas

4 Este ejemplo se lo debo a Carol Rovane. Véase Carol Rovane y Akeel Bilgrami, "Mind, Language, and the Limits of Inquiry", en The Cambridge Companion to Chomsky, ed. James McGilvray (Cambridge: Cambridge University Press, 2005), 181-203.

5 Esto debería matizarse señalando que Chomsky, al final de este capítulo, discute uno de los argumentos de Peirce que hace referencia a consideraciones biológicas, concretamente consideraciones evolutivas basadas en la selección natural (que considera completamente erróneas). Esto indicaría que el propio Peirce era un tanto ambiguo acerca de si tener o no en cuenta su afirmación metodológica relativa a las hipótesis admisibles y a los límites sobre las mismas como dependientes de nuestra biología. 
e ignorando la falta de un conocimiento subyacente más profundo que, de tenerlo, daría sentido a lo que, según este reconocimiento por su parte (y por la de otros) se describía como una fuerza "oculta". Bastaba elaborar teorías inteligibles sobre el mundo. Y para ello no era necesario considerar el mundo inteligible en el sentido más profundo que frustran nuestros límites cognitivos.

Pensadores posteriores -en especial Priestley se presenta como un comentarista extraordinariamente inteligente y lúcidoenunciaron de forma explícita este aspecto metodológico y extrajeron consecuencias sobre cuestiones de la filosofía de la mente que incomodan en la actualidad a los filósofos, pero que, para obtener todo lo que Priestley tenía que ofrecer, tendrían que replantearse lo que presentan como el problema de la mente y el cuerpo o el "complejo problema" de la consciencia. Los filósofos tienen tendencia a clasificar algunos temas como excepcionalmente "complejos" y permanecen satisfechos con esa denominación. Chomsky apela precisamente a esta historia para mostrar, en primer lugar, que no hay nada excepcional en encontrar algo "complejo" de este modo. Así, por ejemplo, lo que la introducción de la "gravedad" supuso para la física se consideró igual de complejo después de Newton, incluso por el propio Newton 6 . La importancia de esto en relación con el llamado problema de la mente y el cuerpo es que pone en duda que -después de Newton- pudiera siquiera seguir siendo formulado de manera coherente. La inicialmente preocupante introducción de algo "misterioso" como la "gravedad" acabó siendo esencial para nuestra concepción de los cuerpos materiales y su mutua interacción sin contacto, de modo que simplemente se incorporó a la ciencia; de hecho, a la nueva lógica científica. De aquí, en cualquier caso, deberíamos llegar a la conclusión filosófica de que todo es inmaterial, de manera que de un problema de la mente y el cuerpo no puede sacarse nada en claro. En una memorable y elocuente alteración de la frase de Ryle, Chomsky dice que, lejos de haber enviado al fantasma al olvido, la máquina fue desechada y el fantasma permaneció intacto. Por lo que respecta a la consciencia, la tendencia del filósofo a requerir que gran parte de nuestra mentalidad sea consciente, una tendencia explícita en filósofos tan dispares como Quine y Searle, es traída a colación al examinar las operaciones de las capacidades sujetas a normas tanto del lenguaje como de la visión. Chomsky concede especial importancia a esto, ya que gran parte de nuestro pensamiento consciente interactúa con aspectos de la mente ocultos a la consciencia, de modo que limitarse a lo consciente impediría un pensamiento científico incluso a la mente consciente.

Dada su preocupación por una explicación científica, le preocupa también mostrar que algunas formas de concebir el lenguaje $y$, más ampliamente, el pensamiento, no sean científicamente sólidas. Existe, en concreto, una amplia discusión acerca de los elementos atómicos de la computación. Invocando puntos establecidos en el capítulo 1, señala que su denominación como "palabras" y "términos léxicos" es engañosa en la bibliografía, porque -al alimentarse de la interfaz conceptualintencional, que se ha demostrado que es primordial, a diferencia de la interfaz sensoriomotora- no se han elaborado por los procesos de externalización. Para los filósofos es aún más sorprendente la afirmación de que, salvo por algunas excepciones explícitamente estipulativas en las matemáticas y las ciencias, no tienen propiedades referenciales y no debe considerarse que tengan relaciones constitutivas con objetos del mundo exterior que no dependen de la mente. El lenguaje I, que es la única noción de lenguaje explicablemente científica, es, por tanto, totalmente interno. Este punto se analiza a través de un examen de las visiones históricas, como las de Aristóteles y Hume, y mediante un análisis de los ejemplos de tales átomos, que van desde los

$6 \mathrm{Y}$ antes de Newton, el movimiento era considerado “el problema complejo" por William Petty, entre otros. 
relativamente concretos como "casa" o "París" a los relativamente abstractos como "persona" o "cosa". La referencia o la denominación son, tal como muestran esos análisis, demasiado contextuales como para soportar el estudio científico y deberían considerarse relevantes para el uso del lenguaje más que para un aspecto constitutivo del lenguaje mismo. Todo esto nos lleva a una taxonomía diferente a la que encontramos entre los filósofos, relegando casi todo lo que tienen en mente como "semántica" a la pragmática.

Estas conclusiones son relevantes para el tema del origen del lenguaje. Las señales entre animales son provocadas por vínculos directos con objetos del mundo exterior. No se entienden si uno de esos vínculos causales queda fuera, mientras que el acento de la discusión precedente estaba puesto precisamente en demostrar que no existen tales vínculos constitutivos causales con una realidad objetiva de los átomos de la computación humana. Esto aporta más razones para concluir que la clase de criaturas que somos, poseedoras del tipo de capacidades para el lenguaje y el pensamiento que poseemos, debería tener una explicación evolutiva como la presentada en el capítulo 1, en lugar de la que Chomsky, citando a Lewontin en el capítulo 2, describe como la "narración" de la evolución gradual desde las criaturas que nos antecedieron, un tipo de explicación que solamente podría satisfacernos si no prestásemos suficiente atención científica a la naturaleza del fenotipo que se está explicando. En parte también es narración, tal como se cita a Lewontin, por la "mala suerte" de no poder acceder a ninguna prueba en la que basar dichas explicaciones. Están ocultas al acceso cognitivo humano, otra forma de limitación.

Por tanto, los límites de nuestra cognición son inevitables por una serie de razones, entre las cuales destaca tomarse en serio el mero hecho de ser criaturas biológicas. A diferencia de Locke, Priestley, Hume, Russell, Peirce y Lewontin, los cuales se encuentran entre los héroes de este capítulo, Hilbert explícitamente ("No existe en absoluto ningún problema irresoluble") y de manera más implícita gran parte de la filosofía contemporánea niegan que haya misterios, negando así un lugar común basado en este mero hecho. Lo fascinante es que Chomsky, tras presentar todo esto, adopta una interesante combinación de actitudes. Por un lado, la idea misma de los límites cognitivos que nos crea "misterios" a los seres humanos que otros sujetos podrían considerar perfectamente abordables, es una concesión a lo que los filósofos denominan metafísica realista. Como él dice, "Dados los lugares comunes misterianos, lo que me resulta inconcebible no es un criterio que determine lo que puede existir". Pero, por otro lado, siguiendo el ejemplo de Newton, su actitud, una vez admitida, es absolutamente pragmática. El simple hecho de que lo que estudiamos, el mundo, pueda no ser inteligible en última instancia, no significa que deberíamos inhibirnos de esforzarnos por elaborar teorías científicas inteligibles del mundo. Incluso el concepto de la libre acción humana, dice Chomsky, que podría ir más allá de cualquiera de los conceptos que poseemos (trascendencia, determinación y aleatoriedad), puede ser un día abordable científicamente, si bien estamos lejos de ello en la actualidad. Esto es bastante diferente de la actitud de Kant, el cual declaró que la libertad era concebible pero nunca cognoscible. Como Peirce y antes que él Newton, y a diferencia de Kant, Chomsky no quiere que su propio misterianismo y su propia insistencia en los límites de nuestras capacidades cognitivas pongan, como dijo Peirce en una ocasión, "obstáculos en el camino al conocimiento".

El capítulo 3, “¿Qué es el bien común?”, elimina la restricción de nuestras naturalezas, planteadas en términos de capacidades individuales (para el lenguaje y la cognición) y nos considera criaturas sociales, tratando de analizara qué es el bien común y qué acuerdos políticos y económicos lo promueven o lo frustran.

La Ilustración tiene gran importancia en el tratamiento de estas cuestiones, aunque lo que Chomsky tiene en mente por lo que respecta a la Ilustración es muy amplio, incluyendo las conocidas figuras "liberales" 
de Adam Smith ${ }^{7}$ y Mill, así como las de una amplia tradición romántica, como Humboldt y Marx. Y su interpretación también es muy amplia, destacando no sólo la vertiente de Smith, frecuentemente obviada por la mayoría de sus críticos liberales y radicales, así como por sus devotos conservadores, sino también los principios que permiten considerar a la Ilustración como una precursora de la posterior tradición anarquista en Europa y a John Dewey en América.

De hecho, el punto de partida de estas investigaciones es individualista y tiene vínculos con los capítulos anteriores. Incluso dentro de sus límites determinados por la biología, las capacidades creativas que posee cada individuo (y que fueron tratadas en el capítulo 1 en el ámbito concreto del lenguaje) son precisamente el tipo de cosas cuyo pleno desarrollo hace que los individuos florezcan como sujetos. La cuestión social del bien común surge necesariamente cuando uno se pregunta qué tipo de instituciones obstaculizan ese desarrollo del individuó. Los marcos sociales, como el capitalismo, que hace hincapié en que el propio interés, obstaculizan el desarrollo de las capacidades individuales en lugar de fomentarlo. Tanto las intensas condenas de Smith a la división del trabajo que destruye nuestra individualidad creativa, como las duras palabras de Dewey sobre la sombra proyectada por los intereses corporativos en prácticamente todos los aspectos de la vida pública y personal, son invocadas para establecer esto. La tradición del anarquismo (desde Bakunin a Rudolph Rocker y el anarcosindicalismo de la época de la guerra civil española) combina las ideas socialistas con los principios liberales de la Ilustración clásica para crear un ideal -de mano de obra cooperativa, el control por parte de los obreros del lugar de trabajo y los medios de producción y la vida social girando en torno a asociaciones voluntarias- que, de llevarse a cabo, barrería los obstáculos para lograr el objetivo del desarrollo humano procedentes tanto del capitalismo de libre mercado como de las tendencias bolcheviques a una "burocracia roja". Las ideas de Dewey sobre la educación revelan cómo, a diferencia de gran parte de la práctica actual de las instituciones educativas, el objetivo del desarrollo humano puede alcanzarse mejor desde una edad temprana.

Hay descripciones enternecedoras de cómo muchos de esos ideales eran fundamentales para el activismo de una amplia gama de movimientos des base, desde la antigua tradición parlamentaria radical de la Inglaterra del siglo XVII hasta las "chicas de las fábricas" y los artesanos sobre quienes Norman Ware escribió en su ensayo sobre los trabajadores industriales de la tradición estadounidense a los teólogos de la liberación de la tradición católica de Centroamérica. Estas antiguas tradiciones laborales democráticas son comparadas con cierto detalle con una idea diferente de democracia, en una tradición que se inicia en Estados Unidos con las restricciones "aristocráticas" de Madison acerca de quién podía gobernar, las cuales están actualizadas en las ideas del "gobierno de los expertos" de Walter Lippmann, la versión estadounidense del vanguardismo leninista que garantiza -como Chomsky deja claro al examinar los resultados de encuestas sobre diversos temas importantes como la asistencia sanitaria- que lo que la gente quiere casi nunca está presente en el programa de la política "democrática". Esta última concepción de la democracia, desde luego, domina la práctica de las sociedades y gobiernos de gran parte del mundo occidental, Y Chomsky señala encantado que, hasta en el peor de los casos, nunca cesa de reivindicar que persigue el elevado ideal del bien común, mostrando que el bien común es universal de una manera bastante paradójica: se proclama que le corresponde a todo el mundo, a pesar de ser vulnerado en todas partes por quienes dicen representar a todos pero básicamente tratan de lograr los objetivos de unos pocos.

7 Chomsky fue el primero en señalar esta vertiente de Smith hace muchas décadas, una vertiente que ha sido estudiada recientemente en una beca de Emma Rothschild comentada por Amartya Sen. 
Dado el punto de partida fundamental de la creatividad humana y la importancia de poder florecer sin impedimentos, no es de extrañar la tendencia de Chomsky hacia el anarquismo, $\mathrm{y}$ su argumento ha sido siempre, tal como vuelve a mostrar en este texto, declarar que cualquier forma de coerción que lo obstaculice no puede nunca darse por sentada. Requiere una justificación. Todas las organizaciones que tienen poder coercitivo, incluido el Estado, deben estar siempre justificadas. La postura por defecto es que no están justificadas hasta que lo estén y siempre y cuando lo estén. Y, dada la contingencia de las "rocas del capitalismo" (su frase) en todos los rincones del mundo, hay, desde luego, una justificación de una idea de Estado que protege al amplio número de personas empujadas a los márgenes de la sociedad (haciéndose eco del propio Smith, que pensaba que únicamente el Estado podía aliviar la vida de opresión a la que el capital industrial somete a la clase obrera $)^{8}$, cosa muy diferente del Estado real en la mayoría de las sociedades, el cual, según Dewey, obedece a las corporaciones $\mathrm{y}$, al hacerlo, elimina el elemento socialista del anarquismo y permite únicamente el elemento libertario, como resultado de lo cual la democracia se convierte en "neodemocracia" (para adaptarse al "neoliberalismo") en la que, si alguien es víctima de la pobreza, ello se debe, como habría dicho Hobbes, a que es lo que ha escogido. De manera que dar la espalda a esto y justificar que el Estado ofrezca protección a quienes sufren bajo el capitalismo, lejos de contradecir al anarquismo, es una aplicación coherente de sus principios en contingencias históricas, un argumento que Chomsky presenta con una maravillosa metáfora que dice haber tomado prestada del movimiento obrero rural de Brasil y ampliado: la metáfora de una "jaula de hierro" cuyo suelo se trata de ampliar al mismo tiempo que se intenta reducir el poder coercitivo del Estado, aunque la jaula nos proteja de las fuerzas destructoras que hay en el exterior, fuerzas que nos debilitan, empobrecen y marginan, por no mencionar que hacen que nuestro planeta sea inhabitable.

He tratado de resumir lo mejor que he sabido un libro cuya fuerza y complejidad intelectual y cuya amplitud de conocimientos y originalidad no pueden plasmarse en un resumen, de modo que se trata de un ejercicio $u$ un deber que es posible que, al final, no ayuden en absoluto al lecto. Sin embargo, diré, sin reparo alguno, que se ha tratado de una tarea tan placentera e instructiva que lo mejor que podría hacer es pedirle al lector que estudie el libro por sí mismo -no sólo por las cualidades que acabo de mencionar, sino por su absoluta seriedad a la hora de examinar las preguntas más profundas de la filosofía y de la ciencia y, por encima de todo, por su inmensa humanidad.

8 Podría añadirse que hay temas en lo que el Estado puede tener justificación porque puede proteger no solo a los marginados y desfavorecidos, sino a todo el mundo de su locura y fatalidad, temas como los relativos al medio ambiente, por ejemplo, y, más en general, proteger a los ciudadanos de los desechos culturales y de la desolación psicológica (en una palabra, temas de "marginación") de los que adolecen las sociedades capitalistas. 